\title{
Research on Discrimination and Denoising of Weak Gas Leakage in Aircraft Air-Tightness System
}

\author{
Yin Dawei ${ }^{1,2, *}$ and Lin Jun ${ }^{1}$ \\ ${ }^{I}$ College of Instrumentation \& Electrical Engineering, Jilin University, Changchun 130026, P.R. China; \\ ${ }^{2}$ Aircraft System Staff Room, Aviation University of Air Force, Changchun 130022, P.R. China
}

\begin{abstract}
Under the environment of complex electromagnetic interference at the airport, high frequency weak signal produced by gas leakage of aircraft air-tightness system, can't be accurately judged alone by testing instrument. For better developing useful signal discrimination, and improving testing effect of gas leakage signal, in the paper, wavelet threshold denoising algorithm and chaos system are applied. Through revising Donoho wavelet threshold denoising algorithm, defects of traditional denoising methods, like lower signal reconstruction precision, additive vibration and so on, are avoided. Signal to noise ratio of useful signal is further advanced. Through improving nonlinear recovery force item of Duffing oscillator equation, effective discrimination of high frequency weak signal is realized under the background of strong noise.
\end{abstract}

Keywords: Duffing oscillator equation, gas leakage, signal discrimination, wavelet threshold denoising.

\section{INTRODUCTION}

Aircraft air-tightness system is an important subsystem, which should be periodical or emergency detected and ensure passenger life safety and normal fly. Gas leakage characteristics of aircraft air-tightness system, are coincident with ultrasonic $40 \mathrm{KHz}$ frequency gas leakage property of small orifice [1].

At present, there are many testing methods on gas leakage of aircraft air-tightness system, meanwhile, a kind of advanced testing technology is to utilize ultrasonic instrument to search gas leakage signal. But, hardware systems of ultrasonic testing instrument have weak antielectromagnetic interference ability, testing effect of gas leakage signal is bad under the background of strong noise, denoising and discrimination of gas leakage signal should be developed by other assistant means [2-4].

A kind of effective signal denoising tool is wavelet denoising in the field of signal processing. According to property of wavelet decomposition, wavelet threshold denoising method divides wavelet coefficients of useful signal and noise signal by threshold mode, possesses prominent noise suppression ability, and keeps characteristics of the extreme value points of original signals. Chaos theory is an important achievement of nonlinear science. Chaos system has strong sensibility to period disturbance and prominent superiority to test weak period signals under the strong noise background, can judge including-noise signal by system transformation.
However, lower signal reconstruction precision and additive vibration are the defects of traditional Donoho wavelet threshold denoising method, weak accuracy of signal discrimination under the strong noise background is the question of traditional Duffing oscillator system. Therefore, in the paper, two methods above-mentioned are improved, to denoise and distinguish gas leakage signals of aircraft air-tightness system under the strong noise background, to help ultrasonic testing better to serve maintenance of aircraft air-tightness system.

\section{IMPROVED WAVELET THRESHOLD DENOISING METHOD}

In traditional Donoho soft or hard threshold denoising method [5-7], wavelet coefficients of estimated signal required by soft threshold method possess well continuity, but wavelet coefficients denoised front and back will produce constant deviation, and descend reconstruction precision of signal. Estimated signal required by hard threshold method can produce additional vibration, and lower smooth degree of reconstruction signal. So, denoising effect of traditional Donoho soft or hard threshold denoising method isn't ideal.

To including noise signal $f(t)=s(t)+n(t)$, target of wavelet threshold denoising is, to eliminate data correlation with the extreme degree, and energy of useful signal is concentrated on bigger wavelet coefficients, noise energy averagely distributes in the whole wavelet field. Through threshold function is reasonably designed, wavelet coefficients corresponding to noise are eliminated, and energy distribution of wavelet coefficients after wavelet decomposition can be 
reflected. Based on this thought, a new threshold function is put forward in the paper.

$$
\hat{w}_{j, k}=\left\{\operatorname{sgn}\left(w_{j, k}\right)\left[\left|w_{j, k}\right|-\left(1-\frac{1}{\exp \left(\frac{\alpha}{\left|w_{j, k}\right|}\right)}\right) \lambda\right],\left|w_{j, k}\right| \geq \lambda ;\right.
$$

To formula $1, w_{j, k}$ represents wavelet coefficient before denoising; $\lambda$ represents threshold value; $\hat{w}_{j, k}$ represents wavelet coefficient after denoising; $\alpha$ is arbitrarily normal constant. When $\alpha$ is sure, formula 1 can keep continuous and smooth state. Moreover, according to formula 1, repression of including noise signal may be written as formula 2

$$
f(x)=\operatorname{sgn}(x)\left(|x|-\left(1-\frac{1}{\exp \left(\frac{\alpha}{\frac{|x|}{t}-t}\right)}\right) t\right),|x| \geq t
$$

Then,

$$
\begin{aligned}
& \lim _{x \rightarrow+\infty} \frac{f(x)}{x}=\lim _{x \rightarrow+\infty} \frac{|x|-\left(1-\frac{1}{\exp \left(\frac{\alpha}{\frac{|x|}{t}-t}\right)}\right) t}{x}=1 \\
& \lim _{x \rightarrow-\infty} \frac{f(x)}{x}=-\lim _{x \rightarrow-\infty} \frac{|x|-\left(1-\frac{1}{\exp \left(\frac{\alpha}{|x|}\right)}\right) t}{x}=1 \\
& \lim _{x \rightarrow \infty}(f(x)-x)=0
\end{aligned}
$$

Above-mentioned formula deduction points out, with wavelet coefficients of including noise signal increasing, threshold function improved in the paper can reduce deviation of wavelet coefficients denoised front and back. Furthermore, if data range of parameter $\alpha$ is extended, then
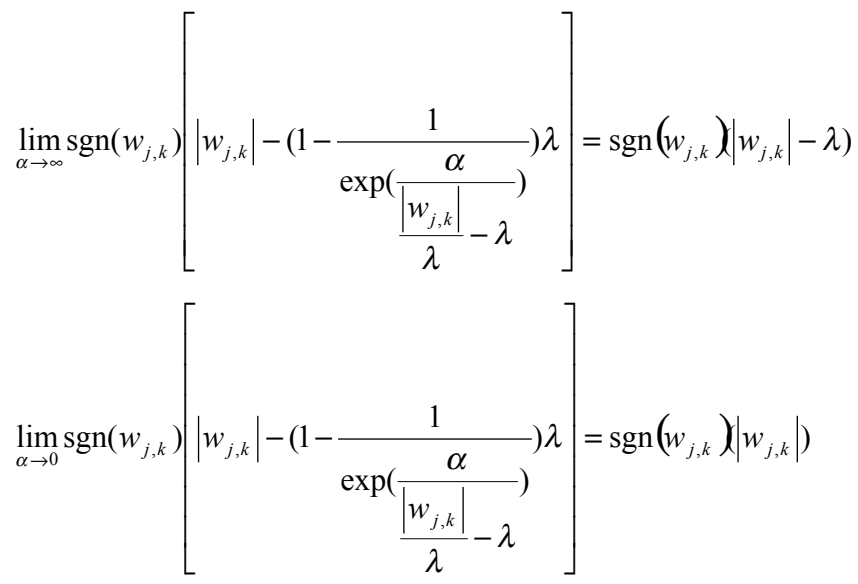

That is to say, when $\alpha \rightarrow \infty$, formula 2 has the same expression with that of soft threshold function. When $\alpha \rightarrow 0$, formula 2 has the same expression with that of hard threshold function. Therefore, through parameter $\alpha$ is properly regulated, threshold function improved in the paper can not only keep smoothness of reconstruction signal, but also avoid constant deviation phenomenon.

\section{IMPROVED CHAOS DUFFING OSCILLATOR EQUATION}

Traditional Duffing oscillator equation is a better tool to distinguish weak period signal. But, this equation is sensitive to statistics property of oscillator system, strong noise, signal characteristics, and can't accurately analyze signal property. So, many scientific researchers do plenty of works to improve Duffing oscillator equation.

In the paper, considering nonlinear recovery force characteristic of chaos system, as well as property of high frequency weak signal in aircraft air-tightness system, nonlinear recovery force of traditional Duffing oscillator equation will be changed into $\alpha x^{5}(t)-\beta x^{7}(t)$, meanwhile, $\alpha=1, \beta=1$. Adding including noise weak period signal into chaos system, expression of Duffing oscillator equation will be changed into,

$$
\frac{1}{\omega^{2}} \ddot{x}(t)+\frac{k}{\omega} \dot{x}(t)+\alpha x^{5}(t)-\beta x^{7}(t)=\gamma_{d} \cos \omega t+a \cos (\omega t)+n(t)
$$

Or it's written as,

$$
\left\{\begin{array}{l}
\dot{x}=\omega y \\
\dot{y}=\omega\left(-k y+a x^{5}-\beta x^{7}+\gamma_{d} \cos (\omega t)+a \cos (\omega t)+n(t)\right)
\end{array}\right.
$$

To formula $3, \dot{x}$ and $\dot{y}$ represent state variable; $k$ represents damp ratio; $\gamma_{d}$ represents chaos threshold; $\omega$ represents period driving force frequency; $a \cos (\omega t)+n(t)$ represents including noise weak period signal, $n(t)$ represents noise.

\subsection{Discrimination Comparison on Weak Period Signal of Different Duffing Oscillator Equations in the Strong Noise Background}

Aiming to traditional Duffing oscillator equation and improved Duffing oscillator equation in the paper, Gaussian white noise with zero mean value and variable noise standard deviation is joined into chaos system, noise standard deviation is changed with 0.05 interval in the range of 0 to 0.5 , variation of chaos threshold with different noise standard deviation is shown in (Fig. 1).

Fig. (1) indicates, when noise standard deviation is lesser, chaos thresholds of two kinds of chaos systems change both smaller, noise variation influences chaos system smaller. When noise standard deviation increases up to a certain 
degree, chaos thresholds of two kinds of chaos systems change both bigger, noise variation influences chaos system bigger. By contrast, in the same variation range of noise standard deviation, chaos threshold of traditional chaos system changes more obvious than that of improved chaos system in the paper. Document [8] emphasized, noise variation will influence critical state of chaos system, even changes critical state of chaos system. That is to say, with noise continuously strengthened, chaos critical state of chaos system will constantly change with chaos thresholds. Compared with traditional chaos system, chaos thresholds of improved chaos system in the paper change in a smaller range. Consequently, improved chaos system in the paper is better means to test weak period signal under the background of strong noise.

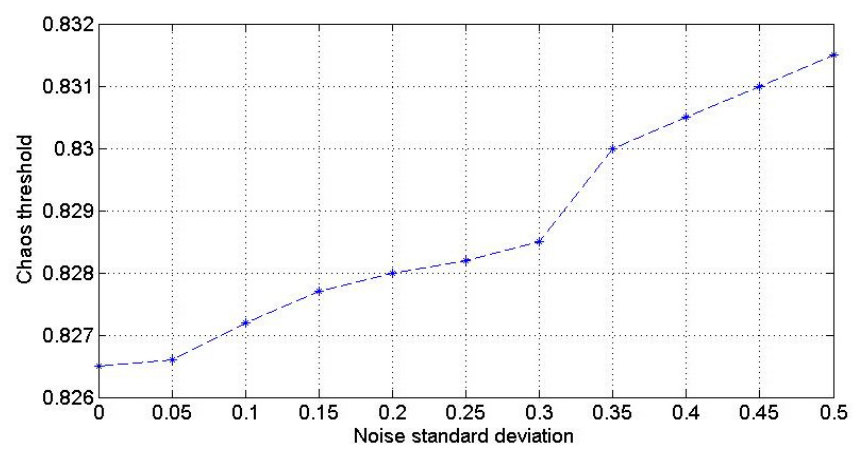

(a) Traditional method

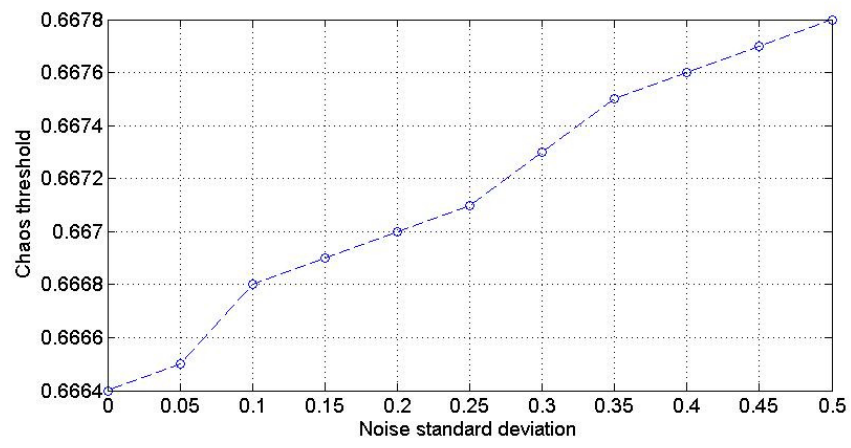

(b) Improved method in the paper

Fig. (1). Relational graph on chaos threshold of two kinds of chaos systems changing with noise standard deviation.

\subsection{Testing Capacity Analysis on High Frequency Weak Period Signal}

Separately, traditional Duffing oscillator equation and improved Duffing oscillator equation in the paper, are added weak period signal $0.0006 * \cos (40000 * t)$ including Gaussian white noise with zero mean value and 0 or 0.15 noise standard deviation, and then observes phase track states of two kinds of chaos systems. The results are shown in the Fig. (2).
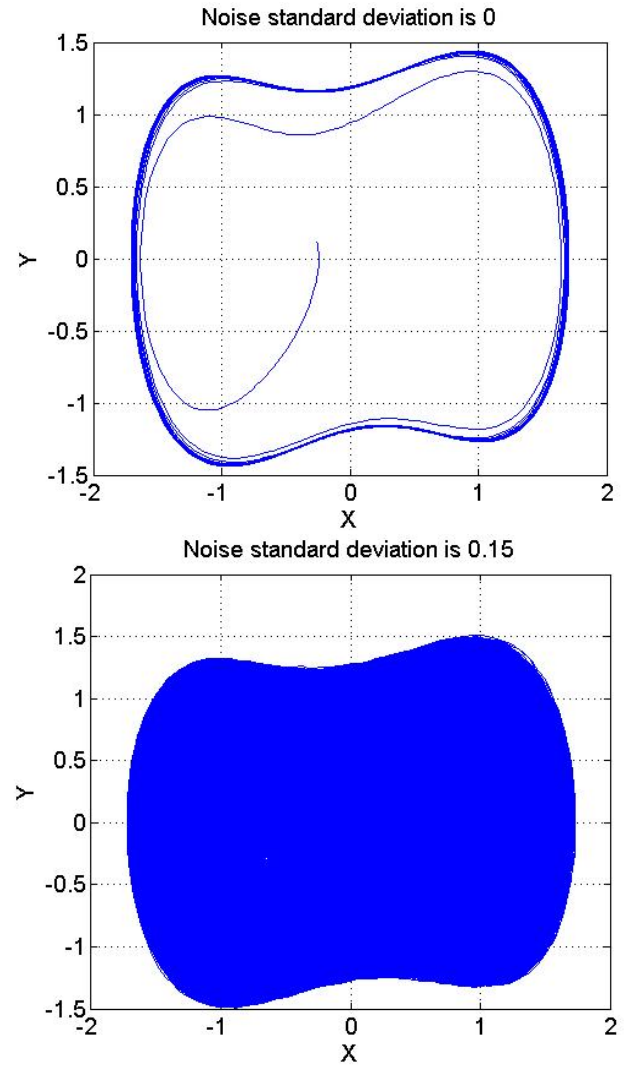

(a) Traditional method
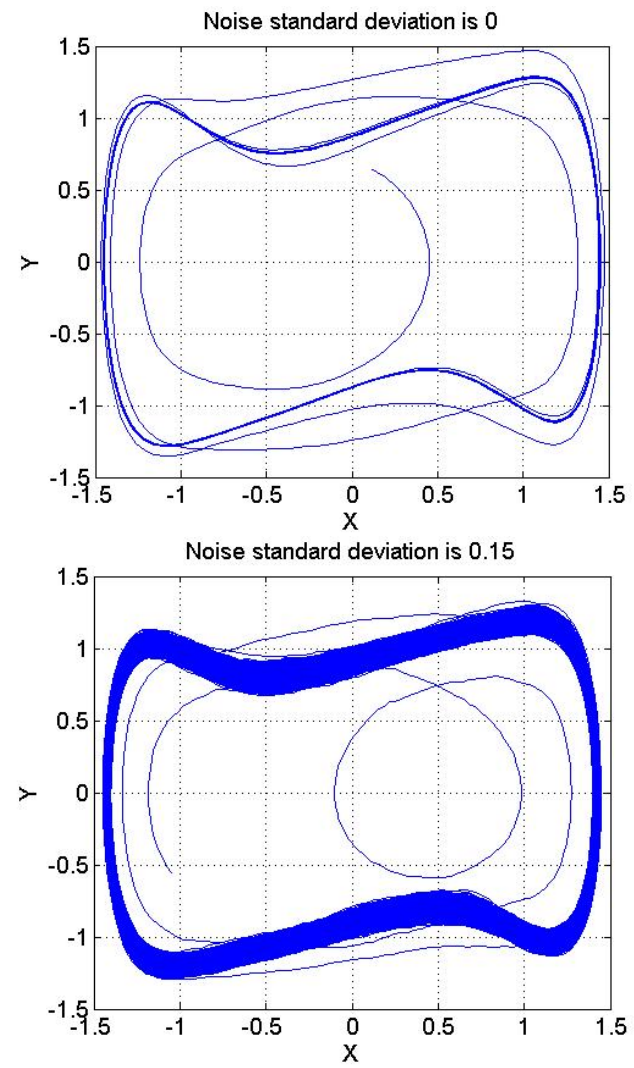

(b) Improved method in the paper

Fig. (2). Phase track diagrams of two kinds of chaos systems, when noise standard deviation is 0 and 0.15 . 
When weak period signal is added into chaos system, practical chaos threshold of system transformation is consist of chaos critical state threshold and amplitude of weak period signal. Figs. (1 and $\mathbf{2})$ reveal, in the invariant condition of chaos critical state threshold, when noise standard deviation is zero, phase tracks required by two kinds of chaos systems both get into large scale period state. When noise standard deviation is 0.15 , under the influence of strong noise, practical chaos threshold of traditional method is more than summation of chaos critical state threshold and amplitude of weak period signal, phase track of chaos system will return chaos state and can't be used to test weak period signal. Nevertheless, practical chaos threshold of improved method in the paper is less than summation of chaos critical state threshold and amplitude of weak period signal, phase track of chaos system keep large scale period state, capacity on testing weak period signal is still useful.

Above-mentioned analysis indicates, with noise continuously strengthened, compared with traditional method, capacity on testing weak period signal of improved method in the paper will be better. One hand, noise intensity is bigger, nonlinear characteristics of chaos system are more obvious. On the other hand, with period driving force frequency changing from low frequency to high frequency, nonlinear characteristics of chaos system are strengthened in a certain degree. Hence, chaos Duffing oscillator equation with high order nonlinear recovery force will satisfy nonlinear characteristics demand of chaos system, and be better fit to distinguish high frequency weak period signal under the strong noise background.

\section{ANALYSIS OF PRACTICAL EXAMPLE}

During the course of gas leakage inspection of aircraft air-tightness system, weak gas leakage signal can't be precisely tested alone by ultrasonic instrument, because plenty of electrical equipments work with strong electromagnetic interference. So, improved wavelet threshold denoising method and improved chaos system in the paper are used, to help ultrasonic instrument to realize signal denoising and discrimination. According to this thought, principle of signal denoising and discrimination is designed in (Fig. 3).

\subsection{Improved Wavelet Threshold Denoising Method Deals with Practical Signal}

In an airport, gas leakage testing of one type of aircraft air-tightness cockpit is done under the strong noise background. Hardware sample signals required by ultrasonic instrument are shown in (Fig. 4). From Fig. (4), it can be found that, useful signal form is hardly observed because of strong noise interference.

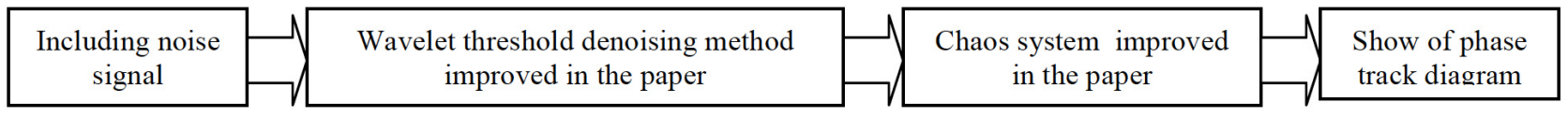

Fig. (3). Principle on common testing weak gas leakage signal by wavelet threshold denoising method and chaos duffing oscillator system.

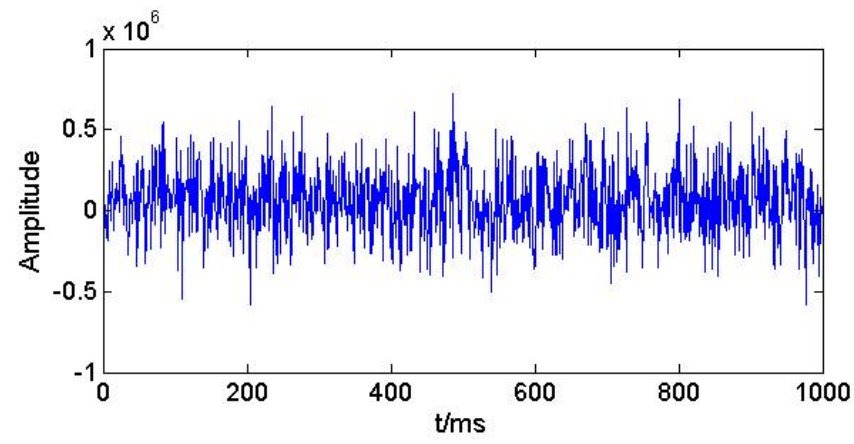

Fig. (4). Hardware sample signals of aircraft air-tightness cockpit required by ultrasonic testing instrument.

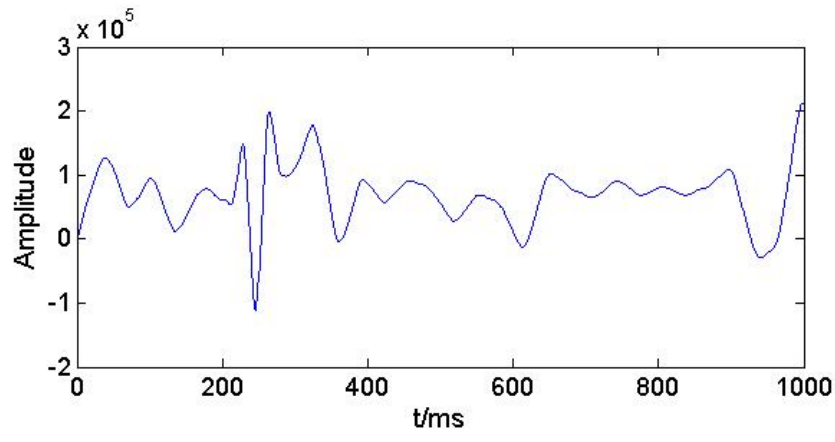

Fig. (5). Denoising effect of wavelet threshold denoising method improved in the paper. 
By use of wavelet threshold denoising method improved in the paper, hardware sample signals in Fig. (4) are denoised, the result is shown in (Fig. 5).

Fig. (5) indicates, gas leakage signals of aircraft airtightness cockpit are better reconstructed by wavelet threshold denoising method improved in the paper, burr phenomena formed by noise are eliminated, barely singular points occur in the denoising signals. When denoising signals are analyzed from time domain to frequency domain, amplitude-frequency characteristic curve will be obtained, as shown in Fig. (6).

Fig. (6) shows, signal amplitude of the $100^{\text {th }}$ sample point is strongest, after the $250^{\text {th }}$ sample point, signal amplitude approaches zero. According to performance index of ultrasonic testing instrument used in the work, the 100th sample point is corresponding to $40 \mathrm{KHz}$ frequency signal, and the $250^{\text {th }}$ sample point is corresponding to $98 \mathrm{KHz}$ frequency signal. That means, more than $98 \mathrm{KHz}$ frequency signals have been wholly filtered, wavelet threshold denoising method improved in the paper better eliminates high frequency noise in hardware sample signals. Besides, by use of denoising estimation indexes, such as signal to noise ratio and root-mean-square error, denoising effect can be directly judged. As shown in Table 1.

Table 1 reveals, in the strong noise background, compared with hardware denoising signals, denoising signals of wavelet threshold denoising method increases signal to noise ratio up to $20 \mathrm{db}$, depresses root-mean-square error up to 4659.2734. Thus, wavelet threshold denoising method improved in the paper possesses better capacity on denoising and extracting useful signals.

\subsection{Improved Chaos Duffing Oscillator System deals with Practical Signal}

After wavelet threshold denoising method deals with hardware sample signals, signal frequency range will be limited between $10 \mathrm{KHz}$ and $100 \mathrm{KHz}$, useful signal is still under the strong noise background. Next, for searching $40 \mathrm{KHz}$ frequency weak gas leakage signal, chaos Duffing oscillator system improved in the paper should be used to develop discrimination work.

Firstly, Duffing oscillator equation with $40 \mathrm{KHz}$ period driving force frequency is constructed, then, chaos system is regulated into critical period state in the condition of none noise and none testing signals, meanwhile, chaos critical threshold $\gamma_{\text {critical }}$ of chaos system is required. As shown in (Fig. 7).

At this time, adding hardware sample signals denoised by wavelet threshold denoising method in the paper into chaos system, you can find, owing to strong noise mixed in hardware sample signals, phase track state of chaos system will change. After front 80 sample points are added into chaos system, chaos system will change from critical period state into chaos state. The result is shown in Fig. (8). After front 100 sample points are added into chaos system, chaos system will change from chaos state into large scale period state. The result is shown in (Fig. 9).

Figs. (8 and 9) show, signal frequency of the $100^{\text {th }}$ sample point is equal with period driving force frequency of chaos system, summation between signal amplitude of the $100^{\text {th }}$ sample point and critical state threshold of chaos system is more than practical chaos threshold of chaos system, so that change chaos system from chaos state into

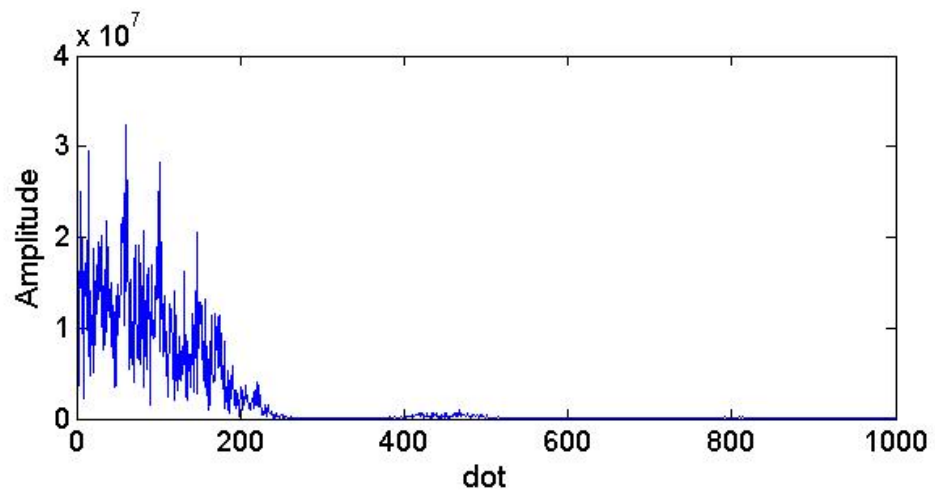

Fig. (6). Signal amplitude-frequency characteristic curve chart required by wavelet threshold denoising method improved in the paper.

Table 1. Comparison on denoising estimation indexes between hardware denoising and wavelet threshold denoising method improved in the paper.

\begin{tabular}{|c|c|c|}
\hline Denoising Estimation Indexes & Hardware Denoising & Wavelet Threshold Denoising Method Improved in the Paper \\
\hline \hline Signal to noise ratio & -21.9571 & 0.1685 \\
\hline Root mean square error & 213959.9650 & 209300.6916 \\
\hline
\end{tabular}




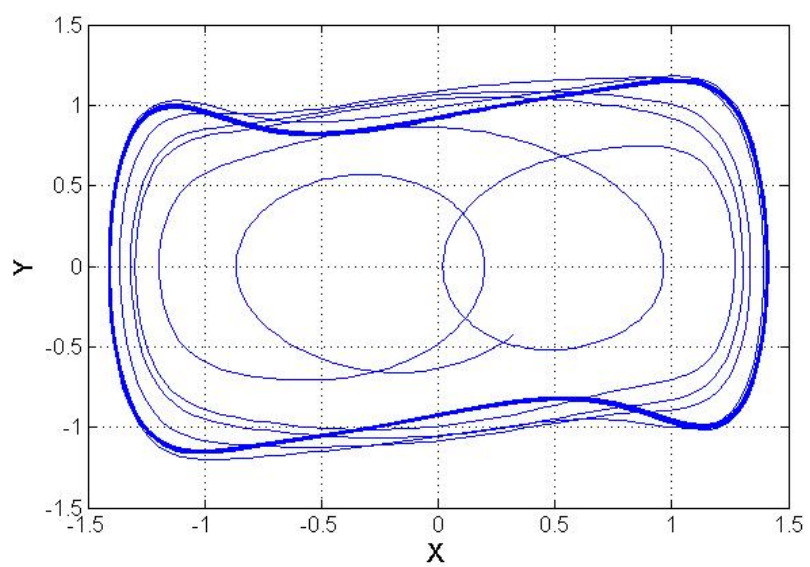

Fig. (7). Phase track diagram of chaos system with $40 \mathrm{KHz}$ period driving force frequency, none noise and none testing signals.

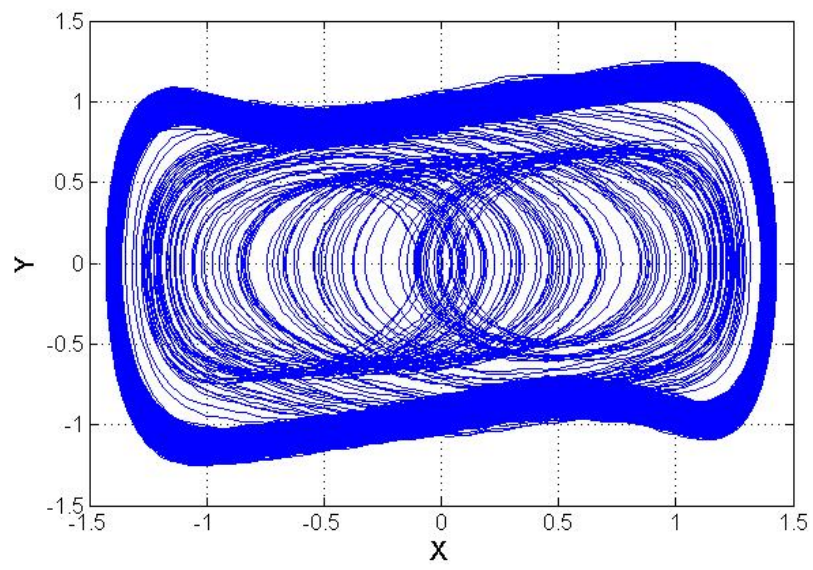

Fig. (8). After front 80 sample points are added, chaos system will be in chaos state.

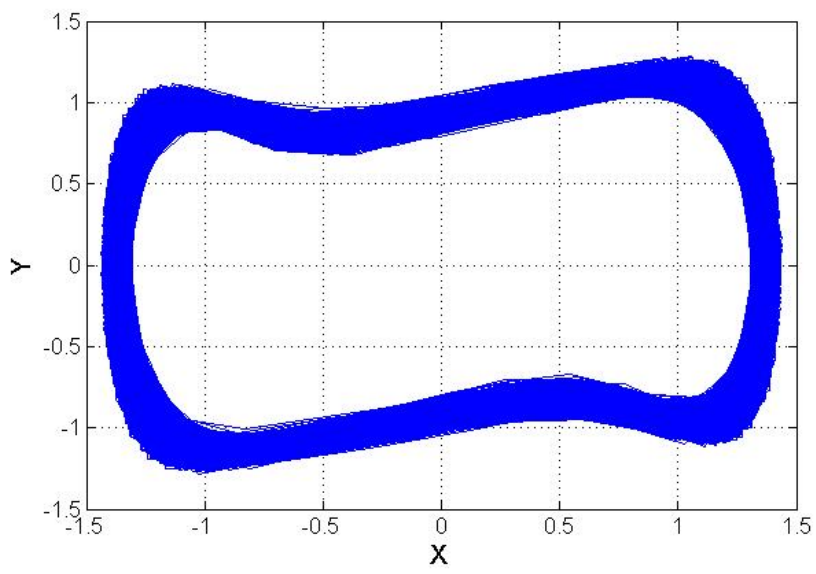

Fig. (9). After front 100 sample points are added, chaos system will be in large scale period state.

large scale period state. That means, the $100^{\text {th }}$ sample point reflects $40 \mathrm{KHz}$ frequency signal, and weak gas leakage signals of the aircraft air-tightness cockpit exist. At this time, once more, chaos system is regulated from large period state into critical period state, chaos threshold $\gamma_{o}$ of chaos system is required, amplitude of weak gas leakage signal is $a=\gamma_{\text {critical }}-\gamma_{o}$. Therefore, according to state transform of chaos system, characteristics of useful gas leakage signal can be gained.

\section{CONCLUSION}

In the paper, according to $40 \mathrm{KHz}$ frequency characteristics of gas leakage of aircraft air-tightness system, and accuracy defect of ultrasonic testing instrument, gas 
leakage signal denoising and discrimination are developed from software level. Firstly, defects of traditional Donoho soft and hard threshold denoising methods are discussed, by formula deduction, wavelet threshold denoising method improved in the paper is testified to be superior. Secondly, considering strong noise background and high frequency signal feature, traditional Duffing oscillator equation is improved, relation between chaos threshold and noise standard deviation is mainly discussed, function of high order nonlinear restore force is emphasized during weak period signal testing of high frequency. Lastly, gas leakage testing of aircraft air-tightness cockpit is exampled, improved wavelet threshold denoising method in the paper and improved chaos Duffing oscillator system are used to deal with weak gas leakage signals, testing effects are practical.

\section{CONFLICT OF INTEREST}

The authors confirm that this article content has no conflict of interest.

\section{ACKNOWLEDGEMENTS}

Declared none.

\section{REFERENCES}

[1] H.X. Cai, Z. Dong, and C. Lin, "Research on air tightness detection method for aircraft gas system", Measurement \& Control Technology, vol. 31, pp. 9-11, 2012.

[2] Y. Zhang, and G.Y. Ji, "Study of weak signal detection based on chaotic oscillator and wavelet theory", Electronic Measurement Technology, vol. 32, no. 6, pp. 40-43, 2009.

[3] L. Satish, and B. Nazneen, "Wavelet-based denoising of partial discharge signals buried in excessive noise and interference", IEEE Transactions on Dielectrics and Electrical Insulation, vol. 10, no. 2, pp. 367-375, 2003.

[4] G.Y. Wang, D.J. Chen, and J.Y. Lin, "The application of chaotic oscillatiors weak signal detection", IEEE Transactions on Industrial Electronics, vol. 46, no. 2, pp. 440-444, 2001.

[5] D.L. Donoho, and I.M. Johnstone, "Ideal spatial adaption via wavelet shrinkage", Biometrika, vol. 81, no. 12, pp. 425-455, 1994.

[6] D.L. Donoho, "De-noising by soft-thresholding", IEEE Transactions on IT, vol. 41, no. 3, pp. 617-643, 1995.

[7] D.L. Donoho, and I. M. Johnstone, "Adapting to unknown smoothness via wavelet shrinkage", Journal of American Statistical Association, vol. 90, no. 432, pp. 1200-1224, 1995.

[8] Y.S. Wang, W.Z. Jiang, and J.J. Zhao, "A new method of weak signal detection using Duffing oscillator and its simulation research", Acta Physica Sinica, vol. 57, no. 4, pp. 2053-2059, 2008.

Received: June 10, 2015

Revised: July 29, 2015

Accepted: August 15, 2015

(C) Dawei and Jun; Licensee Bentham Open.

This is an open access article licensed under the terms of the (https://creativecommons.org/licenses/by/4.0/legalcode), which permits unrestricted, noncommercial use, distribution and reproduction in any medium, provided the work is properly cited. 